\title{
Recent Advances in Lorentz Microscopy
}

\author{
C. Phatak ${ }^{\mathrm{a}}$, A.K. Petford-Long ${ }^{\mathrm{a}, \mathrm{b}}$, M. De Graef ${ }^{\mathrm{c}, *}$ \\ ${ }^{a}$ Materials Science Division, Argonne National Laboratory, 9700 S. Cass Avenue, Argonne, IL \\ 60439, USA. \\ ${ }^{b}$ Department of Materials Science and Engineering, Northwestern University, 2220 Campus \\ Drive, Evanston, IL 60208, USA. \\ ${ }^{c}$ Department of Materials Science and Engineering, Carnegie Mellon University, Pittsburgh, PA \\ 15213, USA
}

\begin{abstract}
Lorentz transmission electron microscopy (LTEM) has evolved from a qualitative magnetic domain observation technique to a quantitative technique for the determination of the magnetization state of a sample. In this review article, we describe recent developments in techniques and imaging modes, including the use of spherical aberration correction to improve the spatial resolution of LTEM into the single nanometer range, and novel in situ observation modes. We review recent advances in the modeling of the wave optical magnetic phase shift as well as in the area of phase reconstruction by means of the Transport of Intensity Equation (TIE) approach, and discuss vector field electron tomography, which has emerged as a powerful tool for the $3 \mathrm{D}$ reconstruction of magnetization configurations. We conclude this review with a brief overview of recent LTEM applications.
\end{abstract}

Keywords: Lorentz transmission electron microscopy, magnetic phase shift, phase reconstruction

${ }^{*}$ Corresponding author. Department of Materials Science and Engineering, Carnegie Mellon University, 5000 Forbes Avenue, Pittsburgh, PA 15213-3890, USA; tel.: +1 412268 8527; fax: +1 4122687596.

Email addresses: cdeanl.gov (C. Phatak), petford. long@anl.gov (A.K. Petford-Long), degraef@cmu.edu (M. De Graef)

Preprint submitted to COSSMS (revised manuscript)

December 30, 2015

(C) 2016. This manuscript version is made available under the Elsevier user license http://www.elsevier.com/open-access/userlicense/1.0/ 


\section{Introduction}

Lorentz transmission electron microscopy (LTEM) is ideally suited to the quantitative analysis of magnetic domain structures at the sub-50 $\mathrm{nm}$ length scale [1]. The ability to image both the microstructure and the magnetic domain structure of an engineering material in the same instrument allows for a direct study of how the often inhomogeneous microstructure plays an important role in influencing the magnetic behavior.

After the early developments by Hale [2] and Boersch and Raith [3], LTEM has seen a steady but slow stream of improvements in both spatial resolution and image quality. In classical terms, high energy electrons traversing a magnetized foil are deflected by the Lorentz force generated by the magnetic induction of the foil. In a quantum mechanical approach, the electron wave passing through the sample is imparted a phase shift which is dependent on the magnetic vector potential of the thin foil. The magnetic vector potential in turn relies on the magnetization of the sample and not on the magnetic induction, and as such LTEM images give direct information about the magnetization state of the sample. It should be noted that the effect of stray fields is also included in these images and has to be treated correctly in interpreting them. For many thin foils the stray fields are minimum and we obtain direct information about the magnetization of the sample; however in magnetic nanostructures, the LTEM images contain integrated information from the magnetization as well as the stray fields. This results in split spots in the diffraction pattern oriented according to the directions of magnetization in the sample, and in magnetic contrast visible in the images. The out-of-focus (Fresnel) mode gives rise to images in which the positions of the magnetic domain walls appear as alternate bright and dark lines, and these can be used to obtain qualitative information. The Fresnel mode is also useful for real-time studies of magnetization reversal (see section 2.3 below) as it is relatively easy to implement, and additionally, a through-focal series of Fresnel mode images forms the input data set from which phase reconstruction of quantitative magnetic induction maps is carried out, as discussed in Section 3.3 below. For the Foucault mode the objective lens is kept in-focus and one of the split spots in the diffraction pattern is blocked by displacing an aperture located coplanar with the diffraction pattern. Only the domains in which the magnetization orientation is such that the electrons are deflected through the aperture, appear bright. The Foucault mode is also qualitative, and is more difficult to implement than the Fresnel mode, because the image contrast depends critically on the lateral position of the blocking aperture and on how close the aperture plane is to the back-focal plane 
of the imaging lens.

For realistic sample thicknesses, the Lorentz deflection angle is typically in the range of tens of micro-radians down to sub micro-radian, i.e., several orders of magnitude smaller than Bragg scattering angles, making it easy to distinguish the two. Direct observation of the structure in the split central diffraction spot using low-angle diffraction (LAD) (or small angle electron scattering (SAES)) provides semi-quantitative information about the magnetic domain structure and can also be used to follow magnetization processes. It should be noted that LAD provides global information from the whole of the illuminated specimen area rather than local information.

Differential phase contrast (DPC) microscopy is a scanning TEM (STEM)based technique. The local Lorentz deflection at the position of the electron probe is determined using an annular quadrant detector sited in the far-field, and the difference signals from opposite segments of the detector provide a direct measure of the two components of the Lorentz deflection, $\beta_{L}$ at each position of the probe across the specimen. The DPC technique requires an increase in instrumental complexity compared with the Foucault and Fresnel imaging modes. In addition, a relatively long time is needed to record each image, meaning that recording images that show changes in magnetization as a function of applied stimuli such as short current pulses in the millisecond time range is not possible unless timedependent effects do not contribute significantly.

In order to obtain quantitative images, one needs to consider the effect of the magnetic material on the phase of the incident electron waves. This wave-optical approach (see Section 3) allows for reconstruction of the phase shift across the image, from which quantitative maps of the magnetic induction can be reconstructed, as is discussed in Section 3.3. Recent advances in aberration correction have lead to improvements in both spatial resolution (down to around $2 \mathrm{~nm}$ ) and in minimum magnetic induction that can be imaged, as will be discussed in more detail below.

A number of review papers and books that include chapters on the theory and practical application of LTEM have been written in the last few years, and these give a good introduction to the classical and wave-optical theories that describe the technique, to the various modes that can be employed, to methods by which magnetic samples can be maintained in a magnetic field-free environment within the microscope, and to the use of in situ experiments to follow magnetization reversal and other processes such as the change in magnetic structure across phase transitions. Examples include chapters in Magnetic Microscopy and its Applications to Magnetic Materials edited by De Graef and Zhu [4], a chapter by Petford-Long 
and Chapman in Magnetic Microscopy of Nanostructures [5] and a recent review article by Petford-Long and De Graef [1].

The focus of the current paper is to review the new advances that have contributed to the development of LTEM, in addition to presenting examples that illustrate recent applications of the technique.

\section{Developments in Techniques and Imaging Modes}

In this section we focus only on developments that extend the techniques beyond those discussed in the review papers cited above.

\subsection{Imaging Modes}

Taniguchi et al. [6] have recently presented a method to obtain Foucault images in a non-dedicated LTEM instrument: the objective lens is switched off to provide a field-free region at the sample, and the condenser lens is then used to bring the electron beam to a cross-over in the plane of the selected area aperture, which is used as the blocking aperture. Taniguchi et al. also note that the same configuration can be used to image low-angle diffraction (LAD) patterns at high magnification.

\subsection{Spherical Aberration-Correction}

In the last few years, the resolution of LTEM images has been greatly increased by the use of spherical-aberration correction [7]. Objective lenses for Lorentz TEM have much higher aberrations than standard TEM lenses (spherical aberration, $C_{s}$, values of 50-8000 mm, and chromatic aberration, $C_{c}$, of 20-40 $\mathrm{mm}$ versus values of 1-2 $\mathrm{mm}$ for $C_{s}$ and $C_{c}$ for a high resolution lens). These high values, combined with the fact that LTEM images for Fresnel microscopy and for phase reconstruction are recorded with very high defocus values, substantially limits the spatial resolution that can be achieved for most applications to 5-10 nm. Phatak et al. reported the use of a $C_{s}$ corrector on a JEOL $2100 \mathrm{~F}$ microscope fitted with a dedicated Lorentz lens, to obtain high spatial and phase resolution images of magnetic monopole defects at the vertices within an artifial spin ice, which had previously not been obtainable. The corrector enabled the $C_{s}$ coefficient to be reduced from $120 \mathrm{~mm}$ to $0.01 \mathrm{~mm}$, resulting in a reduction in the information limit of the microscope from $0.73 \mathrm{~nm}$ to $0.43 \mathrm{~nm}$, together with the ability to reduce the defocus needed for phase reconstructions from $36 \mu \mathrm{m}$ to 19 $\mu \mathrm{m}$ (see Section 3.3 below). 
More recently, McVitie et al. [8] reported results from a spherical aberrationcorrected STEM, which has been further improved with the addition of an eightsegment detector, to enable collection of DPC images with a spatial resolution of around $1 \mathrm{~nm}$. The authors comment on the challenges associated with the small Lorentz deflection angles that are subtended by thin TEM samples with low magnetic induction, and on the possibility of using a pixelated detector, after the method reported by Pennycook et al. in which an image of the diffraction disk is recorded at each position of the probe as it is scanned across the sample, and the image is then processed to extract the magnetic contrast [9].

Chromatic aberration correction has not yet been applied to Lorentz TEM, however the main advantage is that it would allow analysis of thicker specimens. This is extremely important when imaging bulk magnetic materials, because of the change in magnetostatic energy terms associated with making a thin TEM sample from the bulk. In addition, correction of $C_{c}$ would enable a lens with a larger pole-piece gap to be used, thus increasing the space available for in situ stages.

\subsection{Imaging Magnetization Reversal Behavior}

Obtaining a full understanding of the magnetic behavior of a material relies on being able to image specimens in their as-grown state, in remanent states, in the presence of applied fields or currents, and as a function of temperature. From these can be derived basic micromagnetic information, together with features such as the domain wall nature, the presence of domain wall nucleation and pinning sites, and the nature of magnetic phase transitions. In addition, the effects of materials processing (for example, patterning into small elements) on magnetic domain structure and magnetization reversal mechanisms can be analyzed.

In situ magnetic fields can be applied, which enables the local magnetization reversal of a sample to be followed in real-time. A number of approaches have been proposed, including tilting the sample into the field of the objective lens or using a sample holder to which small electromagnets are mounted. Further sophistication can be achieved by combining application of a magnetic field with application of current, as shown by Arita et al. [10] who showed the use of LTEM to image domain walls injected into Permalloy wires using a holder that they had developed. One of the issues associated with in situ applied fields is deflection of the incident electron beam, and although the holder developed by Arita et al. is limited to an applied field of $\pm 200 \mathrm{Oe}$, an advantage of its design is that the deflection of the electron beam by the applied field is compensated by a second set of magnetizing coils mounted on the holder. 
Rodríguez et al. took a different approach, namely to develop a protocol for applying magnetic fields of arbitrary direction and magnitude (up to $4000 \mathrm{Oe}$ ), via the use of the objective lens field and a double-tilt specimen holder in an FEI Titan equipped with a $C_{s}$ corrector [11]. They further presented a Digital Micrograph script that would help control the instrument during the experiments. The application of different field directions was demonstrated by visualizing the motion of domain walls through notched L-shaped Permalloy wires and using these data to construct plots of applied field vs. distance moved for different wire configurations. One point to note is that, as demonstrated in the paper by Rodríguez, use of the lens field to excite a magnetic specimen results in an out-of-plane component of the applied field that can influence the magnetic domain behavior.

Typically the time resolution that can be achieved is of the order of $40 \mathrm{~ms}$, enabling the quasi-static behavior of the magnetization to be visualized. Pollard et al. [12] used a specimen holder excited with a $\mathrm{GHz}$ frequency AC current, to drive the resonant gyrotropic motion of a vortex core in a square Permalloy element, and thus visualize the resonant precession behavior. By measuring the change in resonant gyrotropic precession radius as a function of the magnitude of the applied current, for both clockwise and counter-clockwise precession, a value for the non-adiabatic spin-torque parameter, $\beta$, could be derived. This is typically very difficult to measure experimentally. Imaging of true magnetization dynamics has also been reported, through the use of pulsed probe LTEM in an instrument dedicated to ultrafast imaging [13]. The nucleation and propagation of domain walls in a Ni film following excitation using a laser pulse were observed with a time resolution of a few ns, which is a very exciting development.

\section{Wave-optical approach}

In this section we review briefly the mechanisms of Lorentz microscopy image formation, using a quantum mechanical approach. The basic image formation modalities (Fresnel and Foucault) can be understood based on a simple classical model derived from momentum conservation arguments (e.g., [14]). Such an approach shows that the Lorentz deflection angle, $\theta_{L}$, is two to three orders of magnitude smaller than typical Bragg angles for crystalline materials. The deflection angle for a uniformly magnetized foil of thickness $t$ is given by:

$$
\theta_{L}=\frac{e \lambda}{h} B_{\perp} t=C_{L}(E) B_{\perp} t
$$

where $e$ is the electron charge, $\lambda$ the electron wave length, $h$ Planck's constant, and $B_{\perp}$ the component of the induction normal to the beam. Numerically, the constant 
$C_{L}(E)$ has a value of $0.606426 \mu \mathrm{rad} / \mathrm{T} / \mathrm{nm}$ for $200 \mathrm{kV}$ electrons, so that a $100 \mathrm{~nm}$ foil with saturation induction $B_{\perp}=1 \mathrm{~T}$ will give rise to a deflection of about 60 $\mu \mathrm{rad}$. This small deflection angle justifies the use of a small- $q$ approximation in the wave optical treatment described in the following sections.

\subsection{Theory}

In the quantum mechanical approach, an electron wave experiences a phase shift upon traveling through a region of space with a non-zero electrostatic and/or magnetic vector potential. This phase shift is described by the Aharonov-Bohm expression [15]:

$$
\varphi\left(\mathbf{r}_{\perp}\right)=\varphi_{e}\left(\mathbf{r}_{\perp}\right)+\varphi_{m}\left(\mathbf{r}_{\perp}\right)=\frac{\pi}{\lambda E_{t}} \int_{L} V\left(\mathbf{r}_{\perp}, z\right) \mathrm{d} z-\frac{e}{\hbar} \int_{L} \mathbf{A}\left(\mathbf{r}_{\perp}, z\right) \cdot \mathrm{d} \hat{\mathbf{z}}
$$

where $E_{t}$ is the total beam energy and the integrations are carried out along a straight line $L$ parallel to the incident beam. The vector $\mathbf{r}_{\perp}$ is a position vector in the plane normal to the electron beam. $V$ and $\mathbf{A}$ represent the electrostatic lattice potential and the magnetic vector potential, respectively. The prefactor of the electrostatic integral is also known as the interaction constant; note that the prefactor of the magnetic integral does not depend on the electron energy [16].

For thin specimens and away from strongly diffracting conditions, the sample can be considered to be a strong phase object, i.e., the sample changes only the phase of the electron wave, not the amplitude. For a Lorentz objective lens with point spread function (PSF) $\mathcal{T}\left(\mathbf{r}_{\perp}\right)$, the observed image intensity can be described by the usual convolution expression (ignoring magnification factors):

$$
I\left(\mathbf{r}_{\perp}\right)=\left|\mathrm{e}^{\mathrm{i} \varphi\left(\mathbf{r}_{\perp}\right)} \otimes \mathcal{T}\left(\mathbf{r}_{\perp}\right)\right|^{2} ;
$$

In general, the PSF contains phase changing contributions from the objective lens defocus, different orders of astigmatism, axial coma, and spherical aberration; in addition there are damping contributions due to chromatic aberration and the source spread. In Fourier space, the lens transfer function can be expressed as a simple exponential function of the spatial frequency, $q$, and the lens aberrations:

$$
\mathcal{T}(q)=\mathrm{e}^{-\mathrm{i} \sum_{i=2}^{\dddot{m}} z_{i} q^{i}}
$$

where the complex factors $z_{i}$ represent both the phase shifts and damping con-

tributions of different orders [16]. Since the Lorentz deflection angle is always 
small, the transfer function can be expanded in a Taylor series around $q=0$ to obtain

$$
\mathcal{T}_{L}(q)=1-\mathrm{i} \sum_{i=2}^{\cdots} z_{i} q^{i} .
$$

In the absence of astigmatism and axial coma, the transfer function is determined by the objective lens defocus and spherical aberration. This indicates that spherical aberration, being proportional to $\lambda^{3} q^{4}$, is not a major problem for LTEM observations. However, in a field emission TEM, there is a typically a significant amount of delocalization; this leads to weak diffuse contrast at magnetic domain walls, and therefore a large defocus value is usually required to clearly visible achieve domain wall contrast in LTEM mode. This large defocus, in turn, leads to significant image blurring and hence a relatively low spatial resolution in the range of $10 \mathrm{~nm}$ for the uncorrected LTEM Fresnel mode. Spherical aberration correction has an indirect effect on the LTEM resolution; a reduction of $C_{s}$ leads to a reduction of delocalization, which means that a smaller defocus value can be used to image magnetic domain walls. The smaller defocus means less image blurring and therefore an improved image resolution, potentially down to the single nanometer range.

\subsection{Phase Shift Computation and Image Simulation}

As described in equation 2, the electromagnetic information from the sample is contained in the phase shift of the electron wave. The electrostatic lattice potential is usually approximated by the mean inner potential, $V_{0}$, which is constant throughout the sample, leading to an electrostatic phase shift of $\varphi_{e}=$ $\sigma(E) V_{0} t$, where $\sigma(E)$ depends on the electron energy, and $t$ is the sample thickness. The computation of the magnetic phase shift is more involved since it requires knowledge of the magnetic vector potential. Typically for nanoparticles or patterned nanostructures that are uniformly magnetized, this computation is easily performed using a Fourier space approach by defining a shape function, $D(\mathbf{r})$, equal to 1 inside the object and 0 outside. This approach can be used for regular polyhedral shapes as well as arbitrary shapes $[17,18]$. For non-uniform magnetization, Mansuripur [19] proposed a Fourier space algorithm that assumes that the magnetization is non-uniform only in the $x-y$ plane of the sample and uniform along the $z$ direction; electron beam tilt effects are not easily incorporated in this method. Recently, a new method based on a spherical projection model was proposed by Humphrey et. al. [20]; this model discretizes the magnetization of a given object into a multi-scale grid of voxels represented by a uniformly mag-

netized sphere, thus allowing for the computation of magnetic phase shifts from 
any arbitrary non-uniform magnetization configuration. Additionally, the phase shift can be computed for arbitrary incident beam directions, opening the way to efficient phase shift calculation for tomographic tilt series.

Fresnel and Foucault contrast observed in LTEM images is often non-intuitive or difficult to interpret, especially for nanostructures with complex magnetic domain structures. Thus, image simulations are essential to understand the origin of the observed contrast. The exit electron wave is described by the product of an amplitude factor, which includes absorption and diffraction effects, and a phase factor, which includes electrostatic and magnetic contributions; the resulting image intensity is then given by equation 3. Recently Walton et. al. implemented this approach to simulate LTEM images in an open-source software package called MALTS [21]; their software takes the output file from common micromagnetic simulation software packages, such as OOMMF or MuMax, as input. The LLG Micromagnetic simulator is the only other commercially available software capable of simulating LTEM images [22]. Typically, researchers use their own codes to simulate LTEM images using the above or similar equations along with the paraxial approximation [23-25]. Another approach, recently used by Loudon et. al. [26] to simulate LTEM images of vortices in superconducting $\mathrm{MgB}_{2}$, is based on the Fresnel-Kirchoff integral [27] to compute the wave function as a function of defocus. This approach is in principle equivalent to the paraxial wave approximation.

\subsection{Phase Reconstruction}

To obtain quantitative information from LTEM data, it is necessary to recover the phase of the electron wave. This can be achieved by means of the transport-ofintensity equation (TIE) formalism, which solves the following partial differential equation:

$$
\nabla \cdot\left[I_{0} \nabla \varphi\right]=-\frac{2 \pi}{\lambda} \frac{\partial I}{\partial z}
$$

where $I_{0}$ is the in-focus image intensity and $\partial I / \partial z$ is the longitudinal derivative of the intensity with respect to the beam direction. This equation can be derived from either the transfer function theory of image formation or directly from the Schroedinger equation [4]. In LTEM, the derivative on the right side of the equation is approximated numerically from a through-focus series of images. Solving the equation then leads to the total phase shift, i.e., the sum of the electrostatic and magnetic components. The individual phase shifts can be separated out by: (1) recovering the phase shift for the sample in the upright and flipped (by $180^{\circ}$ ) orientations and then subtracting one from the other, or (2) by varying the acceleration 
voltage to change the prefactor for the electrostatic component and extracting the magnetic component by extrapolation. Once the individual phase shifts are recovered, the quantitative thickness-integrated magnetic induction of the sample can be calculated from the gradient of the magnetic phase shift. Similarly, the electrostatic phase shift can be used to quantify the mean inner potential of the material or to compute the local charge density.

Several methods are available to solve the TIE, such as those based on Fourier transforms [28], using Zernike polynomials [29] or via conjugate gradient methods [30]. Currently the Fourier transform-based method is widely used due to its ease of implementation and fast computation. It has been shown [31] that an appropriate symmetrization of the images before phase reconstruction guarantees a unique reconstruction up to an additive constant. However, since electrostatic and magnetic phase shifts typically have rather different power spectra, using a single boundary condition does not necessarily yield accurate reconstructions. Recent work by Humphrey et al. [32] proposed a modified TIE method that solves for the electrostatic and magnetic phase shifts independently. Computation of the intensity derivative using a truncated Taylor series expansion of the image intensity with respect to defocus was also shown to result in less noisy reconstructions. Tikhonov regularization can be employed to stabilize the phase reconstruction against noise contribution from low frequency components [33]. Other methods, such as total variation regularization, can also be used to improve the TIE solutions [34].

TIE solution methods can also be unstable in the presence of zeros in the in-focus image intensity or zeros in the phase itself; the latter case is true for phase vortices which can be present in electron vortex beam data. A modified TIE method, which takes into account the correct boundary conditions for a phase vortex or phase jumps, has been proposed to solve for the phase under these circumstances [35]. Under additional constraints, the TIE formalism can be modified to obtain the phase from a single defocused image as opposed to an entire through-focus series; Eastwood et al. have used this approach to reconstruct the sample thickness. An interesting connection between the TIE approach and the differential phase contrast technique was recently highlighted by Lubk and Zweck [36].

The electron wave phase shift can also be recovered using in-line and off-axis holography. In-line holography uses an iterative method to solve for the exit wave function in a manner similar to that used for focal series reconstruction in high resolution microscopy [37]. Off-axis holography requires modification of the microscope column by the introduction of an electrostatic biprism that results in an 
interference hologram, which can be used to recover the phase shift. For a comprehensive review of off-axis holography, we refer the reader to a recent article by Linck et al. [38]. Newer methods that involved hybrid approach of incorporating in-line and off-axis holography are also being investigated to combine the benefits from both methods [39].

\section{Vector-Field Electron Tomography (VFET)}

LTEM observations can be used to recover three-dimensional (3D) magnetization information. The electron wave phase shift in equation 2 can be interpreted as a sum of two projection integrals; the electrostatic phase shift is the projection of the 3D scalar electrostatic potential and the magnetic phase shift is the projection of the 3D magnetic vector potential [40]. Thus, using a tomographic tilt series of phase shift data, it is possible to reconstruct the 3D electrostatic and magnetic vector potentials, provided multiple tilt series around orthogonal tilt axes are acquired. A typical VFET experiment then consists of the acquisition of four tilt series, two series about the $x$ and $y$ axes with the sample in the upright orientation, and two more with the sample flipped upside down. Two vector field components can be reconstructed from the two orthogonal tilt series pairs, and the third component is reconstructed by using a divergence condition, such as $\nabla \cdot \mathbf{B}=0$ for the magnetic induction or $\nabla \cdot \mathbf{A}=0$ for the magnetic vector potential (using the Coulomb gauge). The first experimentally reconstructed 3D magnetic vector potential was demonstrated by Phatak et al. [41] for a square Permalloy island.

Subsequently, there have been several efforts to visualize and measure 3D magnetic fields from nanostructures. Yu et al. [42] proposed a three tilt series reconstruction of $\mathbf{A}$ to improve the reconstruction and avoid errors due to singular surfaces; however, this approach is experimentally difficult to implement. Lubk et al. used off-axis electron holography to reconstruct the magnetic stray fields around $\mathrm{Co}_{2} \mathrm{FeGa}$ nanowires [43]. They focused on the stray field by excluding the interior of the sample in their tomographic reconstruction scheme. Tanigaki et al. used a $1 \mathrm{MeV}$ TEM to reconstruct the 3D magnetic spin texture of two coupled Fe discs of $20 \mathrm{~nm}$ thickness [44]. They were able to visualize the core spins of the magnetic vortex structure which were found to point in opposite directions to minimize the interaction energy. Phatak et al. extended iterative reconstruction methods, such as the algebraic reconstruction technique and the simultaneous iterative reconstruction technique, to reconstruct the $3 \mathrm{D}$ magnetic induction in and around magnetic nanostructures [45]; they showed that using iterative methods improves the signal to noise ratio in the reconstructions. Yu et al. [46] reported on 
the application of model-based reconstruction methods to improve the attainable resolution and signal to noise ratio in an attempt to circumvent artifacts due to the limited tilting range of the sample inside the TEM.

\section{Recent Applications of LTEM}

The fact that LTEM requires electron-transparent samples means that it is best applied to nanomaterials such as thin films, patterned nanostructures or nanoparticles, unless the magnetic domain structure is strongly pinned to the microstructure such that creating a thin TEM sample does not affect the bulk domains. For example, Tian et al. [47] reported in situ observations of the changes to microstructure and magnetic domain structure when crossing the martensitic and paramagnetic/ferromagnetic phase transitions in $\mathrm{Mn}_{50} \mathrm{Ni}_{40} \mathrm{Sn}_{10}$. These data could then be used to explain the low temperature bulk magnetization data.

There has been considerable interest in the formation of topological spin structures, one of which is the magnetic skyrmion, in which the magnetization rotates by $180^{\circ}$ from one out-of-plane direction to the other around the boundary of a disk. Skyrmion lattices have been observed to form in bulk magnetic materials such as MnSi over a specific range of temperature and applied field, and Yu et al. [48] used LTEM to visualize the magnetic skyrmion lattice in a MnSi nanowire prepared by thinning MnSi from the bulk. Interest in skyrmions also arises because of the ability to drive their motion with very low currents, giving rise to the potential for their use in information storage applications - so-called 'skyrmionics' [49]. This motion was observed using LTEM in a nanodevice of the helimagnet FeGe at room temperature during in situ biasing, for current densities that are several orders of magnitude lower than that needed to drive domain wall motion in race-track memory structures [50]. The formation of elliptical magnetic bubbles in thinned samples of bulk $\mathrm{La}_{7 / 8} \mathrm{Sr}_{1 / 8} \mathrm{MnO}_{3}$ (LSMO) was reported by Nagai et al. upon application of a high perpendicular magnetic field [51]. LSMO of this composition is a ferromagnetic Mott insulator, and the appearance of the magnetic bubbles was ascribed to a high magnetic anisotropy that is related to orbital ordering. The authors further suggested the possibility of manipulating the bubbles thus formed using electric fields, in the same way as for skyrmions.

In nanostructures of a size as to be single domain, Phatak et al. reported observations of the magnetic frustration around magnetic monopole defects in square artificial spin ice arrays [52] and of the effects of shape anisotropy in Co nanospirals [53]. As the lateral dimension of a patterned nanostructure is increased, so the magnetization configuration changes from single domain to a flux-closure struc- 
ture, with the most commonly observed configuration for a disc being a magnetic vortex in which the magnetization lies in the plane of the disk except at the vortex core, where the magnetic exchange energy forces the magnetization out of the plane. Ngo et al. [54] recently reported a method by which they were able to determine the polarity of the vortex core from the difference of two symmetricallytilted Fresnel mode LTEM images. Phatak et al. [25] reported a novel topological spin structure in coupled magnetic disks of a size at which vortices would be expected in single disks: meron spin structures formed in the two disks in which the magnetization pointed either directly towards or away from the core in each disk. The net magnetization across the coupled disks was thus close to zero and the energy was minimized.

Of interest also are magnetic antidot arrays, in which small regions of a ferromagnetic film are removed, creating pinning sites. Rodríguez et al. explored the behavior of antidot arrays milled into Co thin films with a range of periodicities [55]. For periodicities greater than $300 \mathrm{~nm}$, conventional domain walls were observed running between the pinning sites, but as the periodicity was reduced, so the domain configuration changed such that each domain contained many antidots (refered to as superdomains by the authors).

In the field of therapeutics, superparamagnetic nanoparticles (usually composed of $\mathrm{Fe}_{3} \mathrm{O}_{4}$ ) are being considered for applications as heat mediators in hyperthermia treatment. Of interest is the way in which magnetic dipolar interactions can influence aggregation of the nanoparticles, as this affects the specific power absorption (SPA) and thus the efficiency of the nanoparticles. Campanini et al. [56] have recently used LTEM to show that co-precipitated nanoparticles form large aggregates and thus have a high SPA, when compared with similar-sized nanoparticles synthesized via a thermal decomposition route.

LTEM has also been applied very successfully to the visualization of flux vortex lattices in Type II sperconductors. Loudon et al. showed the importance of careful specimen preparation, with the observation that undulations in the surface of thinned $\mathrm{MgB}_{2}$ created a pinning landscape that strongly controlled the behavior of the vortex lattice [57]. Cottet et al. overcame this problem by careful preparation of the thinned $\mathrm{MgB}_{2}$ specimen and were then able to visualize the expected hexagonal Abrikosov lattice [58]. They related small lattice distortions to impurities that are incorporated during growth.

LTEM offers a unique advantage in the imaging of quasistatic domain behavior in single phase as well as hybrid multiferroics that consist of ferromagnetic and ferroelectric heterostructures. Brintlinger et al. studied the in-situ magnetization reversal of an FeGe thin film deposited on a $\mathrm{BaTiO}_{3}$ substrate under 
application of an electric field. They were able to calculate the stress induced anisotropy in the FeGe film [59]. Hockel et al. also studied magnetization reversal as a function of unipolar and bipolar applied electric field in $\mathrm{Ni}$ thin films on $\left[\mathrm{Pb}\left(\mathrm{Mg}_{1 / 3} \mathrm{Nb}_{2 / 3}\right) \mathrm{O}_{3}\right]_{0.68}-\left[\mathrm{PbTiO}_{3}\right]_{0.32}$ substrate [60]. They were able to observe differences between the two reversals wherein the application of a bipolar electric field resulted in non-reversible magnetic domain wall evolution and Barkhausen jumps.

[Figure 1 about here.]

Figure 1 shows some of the unique advantages of using LTEM to study magnetic nanostructures. Figure 1(a) shows colored magnetic induction maps obtained from a magnetic heterostructure sample with a stack sequence of $\mathrm{NiFe}(20) /$ $\mathrm{Cr}(2) / \mathrm{NiFe}(20) / \mathrm{IrMn}(5)$, patterned into disks, during in situ application of a magnetic field. The color shows the direction of magnetization, as indicated by the color wheel, and the color saturation encodes the magnetization magnitude. The black and white contours in the color map are indicative of the magnetic lines of induction; outside the sample they represent the stray field. The change in the stray field can be seen before and after the jump of the vortex core in the discs. Figure 1(b) shows the magnetization maps obtained from two magnetic nanostructures, namely cobalt nanospirals (top) with a wire diameter of $50 \mathrm{~nm}$ [53], and a chain of $\mathrm{Fe}_{3} \mathrm{O}_{4}$ nano-octahedra (bottom; sample courtesy A. Demortiere, ANL). Figure 1(c) shows the capability of imaging domain walls in functional materials, such as bilayer manganite with a nominal composition of $\mathrm{La}_{2-2 x} \mathrm{Sr}_{1+2 x} \mathrm{Mn}_{2} \mathrm{O}_{7}$ with $x=0.38$. The sample undergoes a ferromagnetic transition at $125 \mathrm{~K}$ and magnetic domain walls can be seen as bright white and dark lines (top). With the aberration correction, the spatial resolution of LTEM is improved over traditional LTEM instruments, so that high resolution images showing the lattice fringes along the $c$ axis in the sample can be observed in LTEM mode (bottom).

[Figure 2 about here.]

Figure 2 illustrates the benefits of VFET to improve our understanding of the 3D nature of magnetic domain walls. Figure 2(a) shows the in-focus image of a TEM sample prepared from an Fe-Pd alloy $\left(\mathrm{Fe}_{68} \mathrm{Pd}_{30} \mathrm{Co}_{2}\right)$. At room temperature, this alloy is in a martensitic state with a strong interaction between the domain walls and twin boundaries formed to accommodate the martensitic transformation [61]. There are two types of domain walls observed in this sample, as seen from 
the color-coded magnetic induction map shown in Figure 2(b): $90^{\circ}$ domain walls which are pinned along the twin boundaries and $180^{\circ}$ domain walls which run in a zig-zag pattern across a twin. Using VFET, the 3D magnetic induction from the TEM sample was reconstructed. Figure 2(c) and Figure 2(d) show the 3D $x$-component of magnetic induction from the dashed boxes shown in Figure 2(b). The color indicates the magnitude of $B_{x}$ (see color bar legend). The 3D reconstructions clearly indicate the inclined nature of the domain walls. From these 3D sections, the angle of the plane of the domain wall (dashed line) with respect to the foil normal (solid line) was measured to be $7^{\circ}$. This measurement was found to be consistent with the diffraction measurements where the $90^{\circ}$ domain walls are known to be on the $\{101\}$ type planes and $180^{\circ}$ domain walls on the $\{100\}$ type planes. This illustrates the powerful capability of the VFET technique to improve our understanding of the 3D nature of domain walls and their correlation with crystallographic orientations by direct visualization of the magnetic induction.

\section{Acknowledgments}

MDG would like to acknowledge the DOE Basic Energy Sciences program DE-FG02-01ER45893 for financial support. AKPL and CP was supported by the U.S. Department of Energy, Office of Science, Basice Energy Sciences Division of Materials Sciences and Engineering. Use of the Center for Nanoscale Materials, supported by the U.S. Department of Energy, Office of Science, Office of Basic Energy Sciences under cotract DE-AC02-06CH11357 is acknowledged.

\section{References}

[1] A.K. Petford-Long and M. De Graef. Characterization of Materials, chapter Lorentz Microscopy, pages 1787-1801. John Wiley \& Sons, 2012.

[2] M.E. Hale, H.W. Fuller, and H. Rubenstein. Magnetic domain observations by electron microscopy. J. Appl. Phys., 30:789-791, 1959.

[3] H. Boersch and H. Raith. Elektronmikroskopische Abbildung Weißscher Bezirke in dünnen ferromagnetischen Schichten. Naturwissenschaften, 46:574, 1959.

[4] M. De Graef and Y. Zhu, editors. Magnetic Microscopy and its Applications to Magnetic Materials, volume 36. Academic Press, 2000. 
[5] A.K. Petford-Long and J.N. Chapman. Lorentz Microscopy. In H. Hopster and H.P. Oepen, editors, Magnetic Microscopy of Nanostructures, chapter 4, pages 67-86. Springer, 2005.

[6] Y. Taniguchi, H. Matsumoto, and K. Harada. Foucault imaging by using non-dedicated transmission electron microscope. Applied Physics Letters, 101(9):093101, 2012.

[7] R. Erni. Aberration-Corrected Imaging in Transmission Electron Microscopy: An Introduction (2nd Edition). Imperial College Press, 2010.

[8] S. McVitie, D. McGrouther, S. McFadzean, D. A. MacLaren, K. J. O’Shea, and M. J. Benitez. Aberration corrected Lorentz scanning transmission electron microscopy. Ultramicroscopy, 152:57-62, 2015.

[9] T.J. Pennycook, A.R. Lupini, H. Yang, M.F. Murfitt, L. Jones, and P.D. Nellist. Efficient phase contrast imaging in STEM using a pixelated detector. Part I: Experimental demonstration at atomic resolution. Ultramicroscopy, 151:160-167, 2015.

[10] M. Arita, R. Tokuda, K. Hamada, and Y. Takahashi. Development of tem holder generating in-plane magnetic field used for in-situ tem observation. Materials Transactions, 55(3):403-409, 2014.

[11] L.A. Rodríguez, C. Magén, E. Snoeck, C. Gatel, L Marín, L. SerranoRamón, J.L. Prieto, M. Muñoz, P.A. Algarabel, L. Morellon, J.M. De Teresa, and M.R. Ibarra. Quantitative in situ magnetization reversal studies in Lorentz microscopy and electron holography. Ultramicroscopy, 134:14454, 2013.

[12] S.D. Pollard, L. Huang, K.S. Buchanan, D.A. Arena, and Y. Zhu. Direct dynamic imaging of non-adiabatic spin torque effects. Nature communications, 3:1028, 2012.

[13] H.S. Park, J.S. Baskin, and A.H. Zewail. 4D Lorentz electron microscopy imaging: magnetic domain wall nucleation, reversal, and wave velocity. Nano letters, 10(9):3796-803, 2010.

[14] M. De Graef. Lorentz microscopy: Theoretical basis and image simulations. In M. De Graef and Y. Zhu, editors, Magnetic Microscopy and its Applications to Magnetic Materials, volume 36 of Experimental Methods in the Physical Sciences, chapter 2. Academic Press, 2000. 
[15] Y. Aharonov and D. Bohm. Significance of electromagnetic potentials in the quantum theory. Phys. Rev., 115:485-491, 1959.

[16] M. De Graef. Introduction to Conventional Transmission Electron Microscopy. Cambridge University Press, 2003.

[17] M. Beleggia and Y. Zhu. Electron-optical phase shift of magnetic nanoparticles I. Basic concepts. Philosophical Magazine, 83(8):1045-1057, January 2003.

[18] M. Beleggia, Y. Zhu, S. Tandon, and M. De Graef. Electron-optical phase shift of magnetic nanoparticles II. Polyhedral particles. Philosophical Magazine, 83(9):1143-1161, January 2003.

[19] M Mansuripur. Computation of electron diffraction patterns in Lorentz electron microscopy of thin magnetic films. Journal of applied physics, 69(4):2455-2464, 1991.

[20] E. Humphrey and M. De Graef. On the computation of the magnetic phase shift for magnetic nano-particles of arbitrary shape using a spherical projection model. Ultramicroscopy, 129:36-41, 2013.

[21] Stephanie K. Walton, Katharina Zeissler, Will R. Branford, and Solveig Felton. MALTS: A tool to simulate lorentz transmission electron microscopy from micromagnetic simulations. IEEE Transactions on Magnetics, 49(8):4795-4800, 2013.

[22] M. R. Scheinfein. LLG Micromagnetics Simulator, 4 edition, 2015.

[23] S. McVitie and M. Cushley. Quantitative Fresnel Lorentz microscopy and the transport of intensity equation. Ultramicroscopy, 106(4-5):423-431, 2006.

[24] Yi Qi, T. Brintlinger, and John Cumings. Direct observation of the ice rule in an artificial kagome spin ice. Physical Review B, 77(9):1-4, March 2008.

[25] C. Phatak, A.K. Petford-Long, and O. Heinonen. Direct observation of unconventional topological spin structure in coupled magnetic discs. Phys. Rev. Lett., 108:067205, 2012.

[26] J. C. Loudon, S. Yazdi, T. Kasama, N. D. Zhigadlo, and J. Karpinski. Measurement of the penetration depth and coherence length of $\mathrm{MgB}_{2}$ in 
all directions using transmission electron microscopy. Physical Review B, 91(5):054505, 2015.

[27] M. Beleggia, G. Pozzi, J. Masuko, N. Osakabe, K. Harada, T. Yoshida, O. Kamimura, H. Kasai, T. Matsuda, and a. Tonomura. Interpretation of Lorentz microscopy observations of vortices in high-temperature superconductors with columnar defects. Physical Review B, 66(17):1-10, 2002.

[28] D. Paganin and K. Nugent. Noninterferometric Phase Imaging with Partially Coherent Light. Physical Review Letters, 80(12):2586-2589, March 1998.

[29] T. E. Gureyev, a. Roberts, and K. a. Nugent. Phase retrieval with the transport-of-intensity equation: matrix solution with use of Zernike polynomials. Journal of the Optical Society of America A, 12(9):1932, September 1995.

[30] Bindang Xue and Shiling Zheng. Phase retrieval using the transport of intensity equation solved by the FMG-CG method. Optik, 122(23):2101-2106, 2011.

[31] V V Volkov, Y Zhu, and M De Graef. A new symmetrized solution for phase retrieval using the transport of intensity equation. Micron (Oxford, England : 1993), 33(5):411-6, January 2002.

[32] E. Humphrey, C. Phatak, A.K. Petford-Long, and M. De Graef. Separation of electrostatic and magnetic phase shifts using a modified transport-ofintensity equation. Ultramicroscopy, 139:5-12, 2014.

[33] Kazuo Ishizuka and Brendan Allman. Phase measurement of atomic resolution image using transport of intensity equation. Journal of electron microscopy, 54(3):191-7, July 2005.

[34] Lei Tian, Jonathan C. Petruccelli, and George Barbastathis. Nonlinear diffusion regularization for transport of intensity phase imaging. Optics Letters, 37(19):4131, 2012.

[35] A. Lubk, G. Guzzinati, F. Börrnert, and J. Verbeeck. Transport of Intensity Phase Retrieval of Arbitrary Wave Fields Including Vortices. Physical Review Letters, 111(17):173902, 2013. 
[36] A. Lubk and J. Zweck. Differential phase contrast: An integral perspective. Phys. Rev. A, 91:023805, 2015.

[37] Christoph T Koch. Towards full-resolution inline electron holography. $\mathrm{Mi}$ cron (Oxford, England: 1993), October 2013.

[38] Martin Linck, Bert Freitag, Stephan Kujawa, Michael Lehmann, and Tore Niermann. State of the art in atomic resolution off-axis electron holography. Ultramicroscopy, 116:13-23, 2012.

[39] C Ozsoy-Keskinbora, CB Boothroyd, RE Dunin-Borkowski, PA van Aken, and CT Koch. Hybridization approach to in-line and off-axis (electron) holography for superior resolution and phase sensitivity. Scientific reports, 4, 2014.

[40] C Phatak, M Beleggia, and M De Graef. Vector field electron tomography of magnetic materials: theoretical development. Ultramicroscopy, 108(6):50313, May 2008.

[41] Charudatta Phatak, Amanda Petford-Long, and Marc De Graef. ThreeDimensional Study of the Vector Potential of Magnetic Structures. Physical Review Letters, 104:253901, June 2010.

[42] Rotha Yu, Michael Morgan, and David Paganin. Lorentz-electron vector tomography using two and three orthogonal tilt series. Physical Review A, 83(2):023813, February 2011.

[43] A. Lubk, D. Wolf, P. Simon, C. Wang, S. Sturm, and C. Felser. Nanoscale three-dimensional reconstruction of electric and magnetic stray fields around nanowires. Applied Physics Letters, 105(17):173110, October 2014.

[44] Toshiaki Tanigaki, Yoshio Takahashi, Tomokazu Shimakura, Tetsuya Akashi, Ruriko Tsuneta, Akira Sugawara, and D Shindo. Three-Dimensional Observation of Magnetic Vortex Cores in Stacked Ferromagnetic Discs. Nano letters, January 2015.

[45] C Phatak and D Gürsoy. Iterative reconstruction of magnetic induction using Lorentz transmission electron tomography. Ultramicroscopy, 150:54-64, December 2015. 
[46] Zhou Yu, J.B. Thibault, C.a. Bouman, K.D. Sauer, and Jiang Hsieh. Non-homogeneous updates for the iterative coordinate descent algorithm. Proceedings of the SPIE/IS\&T Symposium on Computational Imaging V, 6498(1B):1-12, 2007.

[47] H.F. Tian, J.B. Lu, L. Ma, H.L. Shi, H.X. Yang, G.H. Wu, and J.Q. Li. Martensitic transformation and magnetic domains in $\mathrm{Mn}_{50} \mathrm{Ni}_{40} \mathrm{Sn}_{10}$ studied by in-situ transmission electron microscopy. Journal of Applied Physics, 112(3):033904, 2012.

[48] X. Yu, J.P. DeGrave, Y. Hara, T. Hara, S. Jin, and Y. Tokura. Observation of the magnetic skyrmion lattice in a MnSi nanowire by Lorentz TEM. Nano Letters, 13(8):3755-9, 2013.

[49] A. Fert, V. Cros, and J. Campaio. Skyrmions on the track. Nature Nanotechnology, 8:152-156, 2013.

[50] X.Z. Yu, N. Kanazawa, W.Z. Zhang, T. Nagai, T. Hara, K. Kimoto, Y. Matsui, Y. Onose, and Y. Tokura. Skyrmion flow near room temperature in an ultralow current density. Nature Communications, 3:988, 2012.

[51] T. Nagai, M. Nagao, K. Kurashima, T. Asaka, W. Zhang, and K. Kimoto. Formation of nanoscale magnetic bubbles in ferromagnetic insulating manganite $\mathrm{La}_{7 / 8} \mathrm{Sr}_{1 / 8} \mathrm{MnO}_{3}$. Applied Physics Letters, 101(16):162401, 2012.

[52] C. Phatak, A.K. Petford-Long, O. Heinonen, M. Tanase, and M. De Graef. Nanoscale structure of the magnetic induction at monopole defects in artificial spin-ice lattices. Physical Review B, 83(17):174431, 2011.

[53] C. Phatak, Y. Liu, E.B. Gulsoy, D. Schmidt, E. Franke-Schubert, and A.K. Petford-Long. Visualization of the Magnetic Structure of Sculpted ThreeDimensional Cobalt Nanospirals. Nano Letters, 14:759-764, 2014.

[54] D.-T. Ngo and S. McVitie. Visualization of vortex core polarity in $\mathrm{NiFe}$ nanodots by tilted Fresnel images. Ultramicroscopy, 111(8):1276-85, 2011.

[55] L.A. Rodríguez, C. Magén, E. Snoeck, C. Gatel, C. Castán-Guerrero, J. Sesé, L.M. García, J. Herrero-Albillos, J. Bartolomé, F. Bartolomé, and M.R. Ibarra. High-resolution imaging of remanent state and magnetization reversal of superdomain structures in high-density cobalt antidot arrays. Nanotechnology, 25(38):385703, 2014. 
[56] M. Campanini, R. Ciprian, E. Bedogni, A. Mega, V. Chiesi, F. Casoli, C. de Julián Fernández, E. Rotunno, F. Rossi, A. Secchi, F. Bigi, G. Salviati, C. Magén, V. Grillo, and F. Albertini. Lorentz microscopy sheds light on the role of dipolar interactions in magnetic hyperthermia. Nanoscale, 7(17):7717-25, 2015.

[57] J.C. Loudon, C.J. Bowell, N.D. Zhigadlo, J. Karpinski, and P.A. Midgley. Imaging flux vortices in $\mathrm{MgB}_{2}$ using transmission electron microscopy. Physica C: Superconductivity, 474:18-20, 2012.

[58] M.J.G. Cottet, M. Cantoni, B. Mansart, D.T.L. Alexander, C. Hébert, N.D. Zhigadlo, J. Karpinski, and F. Carbone. Quantitative imaging of flux vortices in the type-II superconductor $\mathrm{MgB}_{2}$ using cryo-Lorentz transmission electron microscopy. Physical Review B, 88(1):014505, 2013.

[59] Todd Brintlinger, Sung Hwan Lim, Kamal H. Baloch, Paris Alexander, Yi Qi, John Barry, John Melngailis, Lourdes Salamanca-Riba, I. Takeuchi, and John Cumings. In situ observation of reversible nanomagnetic switching induced by electric fields. Nano Letters, 10(4):1219-1223, 2010.

[60] J. L. Hockel, S. D. Pollard, K. P. Wetzlar, T. Wu, Y. Zhu, and G. P. Carman. Electrically controlled reversible and hysteretic magnetic domain evolution in nickel film/ $\mathrm{Pb}(\mathrm{Mg} 1 / 3 \mathrm{Nb} 2 / 3) \mathrm{O} 3]$ 0.68-[PbTiO3]0.32 (011) heterostructure. Applied Physics Letters, 102(24):2011-2016, 2013.

[61] A. Budruk, C. Phatak, A.K. Petford-Long, and M. De Graef. In situ lorentz TEM magnetization study of a NiMnGa ferromagnetic shape memory alloy. Acta Materialia, 59(12):4895-4906, 2011. 


\section{List of Figures}

1 (a) In-situ behavior in coupled magnetic heterostructure disks consisting of ferromagnetic and antiferromagnetic layers showing the jump of the vortex core as a function of applied field, (b) Magnetization maps in cobalt nanospirals (top) and $\mathrm{Fe}_{3} \mathrm{O}_{4}$ octahedral nanoparticles (bottom), and (c) Magnetic domain wall in bilayer manganite (top) and high resolution image showing the lattice fringes along c-axis obtained using spherical aberration corrected Lorentz TEM. . . . . . . . . . . . . . . . . . . .

2 (a) In-focus image showing an FePd alloy TEM sample. The dashed lines indicate twin boundaries in the material. (b) shows the color-coded magnetic induction map obtained from the sample indicating the presence of two types of domain walls: $90^{\circ}$ along the twin boundaries and $180^{\circ}$ across a twin. (c) and (d) show the $3 \mathrm{D} x$ component of magnetic induction reconstructed using VFET from the two boxed regions in (b). The dashed line indicates the plane of the domain wall and the solid line indicates the foil normal. The angle between the two was determined to be $7^{\circ}$ from 3D measurements. .................. . . 24 


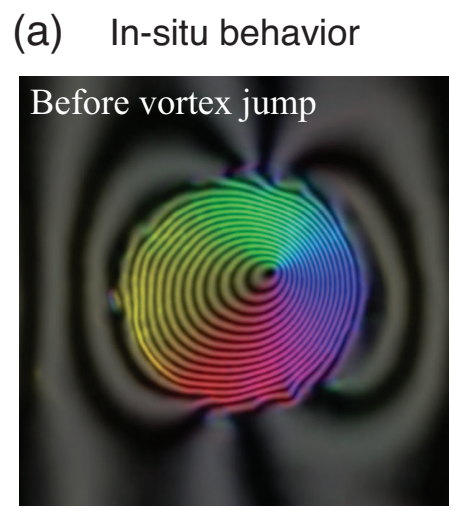

(b) Nanostructures
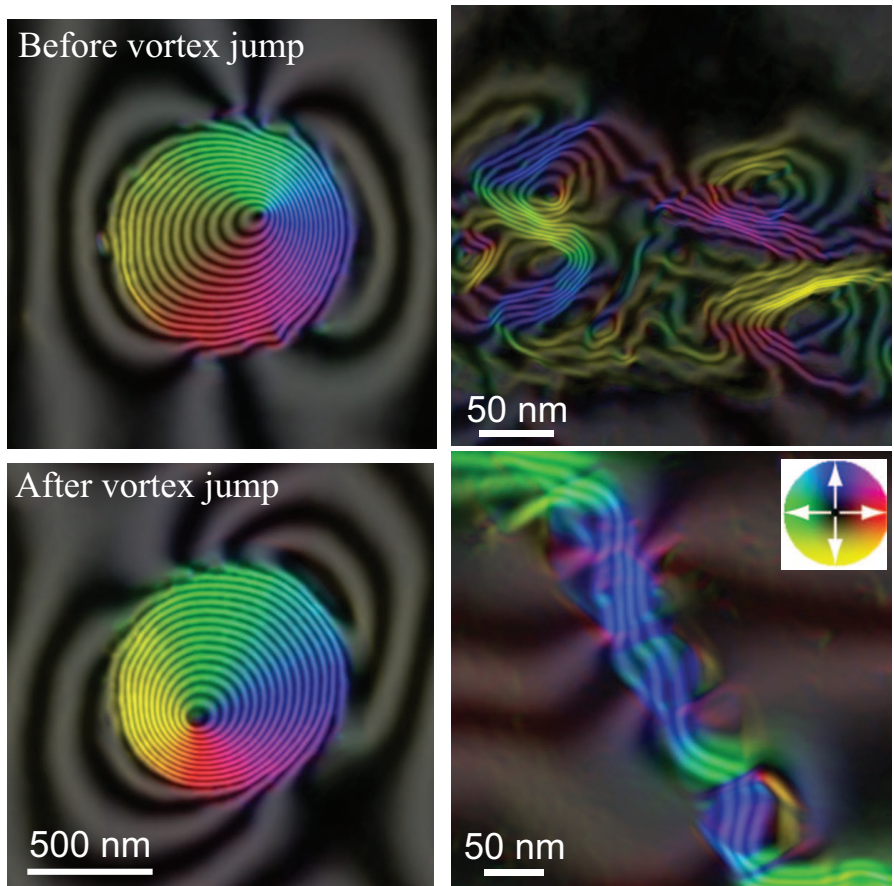

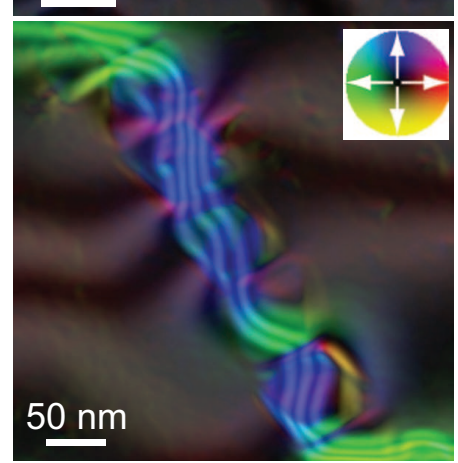

(c) Functional Materials
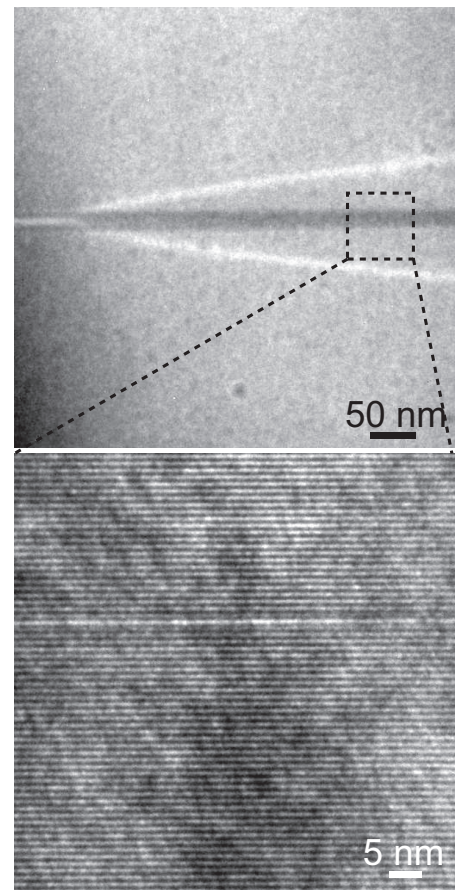

Figure 1: (a) In-situ behavior in coupled magnetic heterostructure disks consisting of ferromagnetic and antiferromagnetic layers showing the jump of the vortex core as a function of applied field, (b) Magnetization maps in cobalt nanospirals (top) and $\mathrm{Fe}_{3} \mathrm{O}_{4}$ octahedral nanoparticles (bottom), and (c) Magnetic domain wall in bilayer manganite (top) and high resolution image showing the lattice fringes along c-axis obtained using spherical aberration corrected Lorentz TEM. 
(a)
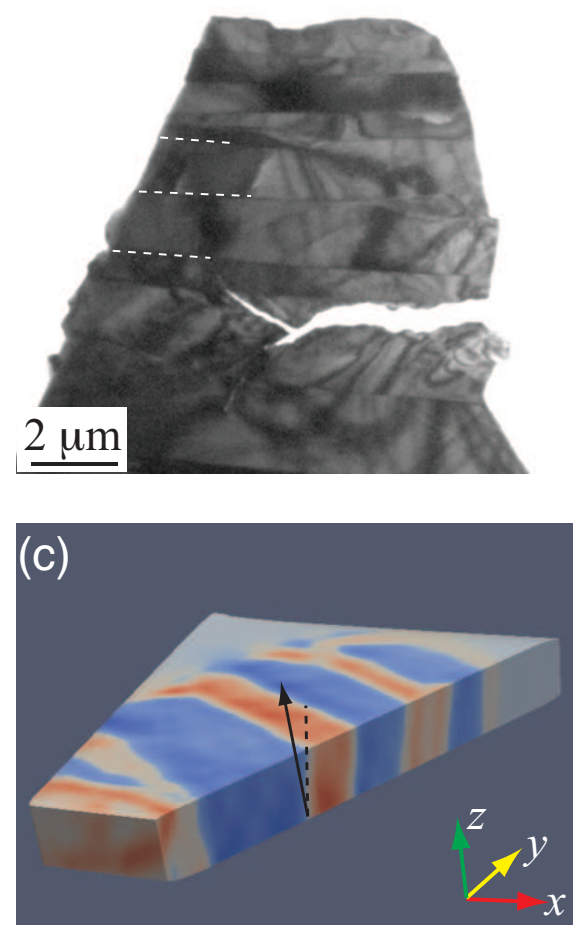
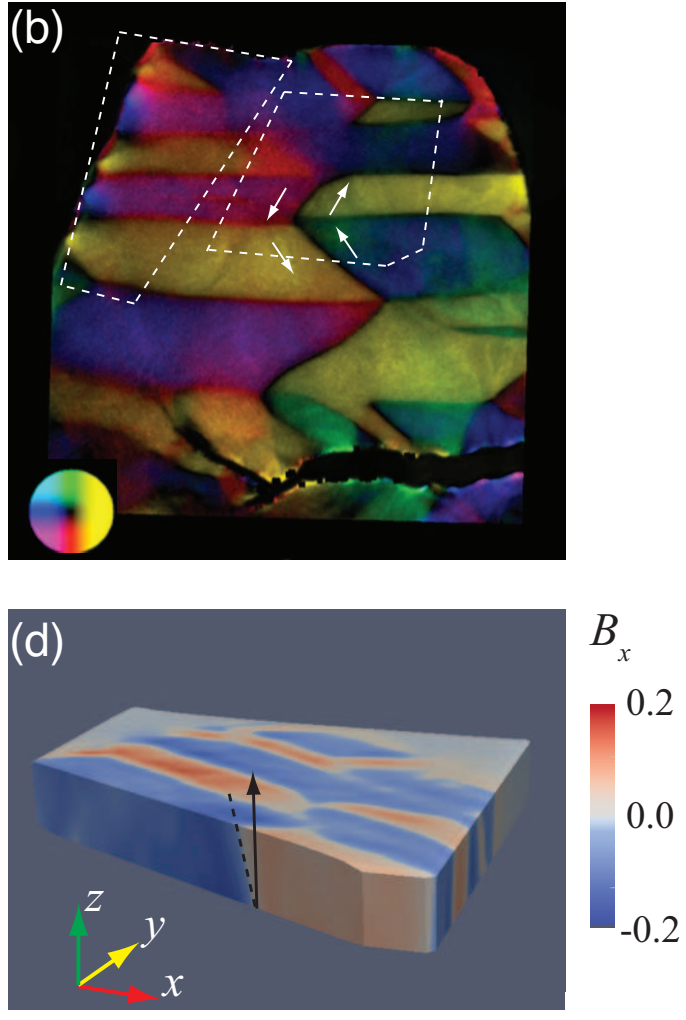

Figure 2: (a) In-focus image showing an FePd alloy TEM sample. The dashed lines indicate twin boundaries in the material. (b) shows the color-coded magnetic induction map obtained from the sample indicating the presence of two types of domain walls: $90^{\circ}$ along the twin boundaries and $180^{\circ}$ across a twin. (c) and (d) show the 3D $x$ component of magnetic induction reconstructed using VFET from the two boxed regions in (b). The dashed line indicates the plane of the domain wall and the solid line indicates the foil normal. The angle between the two was determined to be $7^{\circ}$ from 3D measurements. 